www.jmscr.igmpublication.org

Impact Factor 5.84

Index Copernicus Value: 83.27

ISSN (e)-2347-176x ISSN (p) 2455-0450

crossref DOI: _https://dx.doi.org/10.18535/jmscr/v5i7.46

Journal Of Medical Science And Clinical Research

\title{
Study of Cryptosporidiosis in Children with Hematological and Solid Malignancies Suffering From Diarrhea
}

\author{
Authors \\ Magdy Abd Elfattah Ramadan ${ }^{1}$, Maha Yousef Kamal ${ }^{1}$, Mona Mohamed Tolba ${ }^{2}$, \\ Ekram Abdelsalam Elfaramawy Mohamed \\ ${ }^{1}$ Department of Pediatrics, Faculty of Medicine, University of Alexandria \\ ${ }^{2}$ Department of Parasitology, Faculty of Medicine, University of Alexandria \\ Corresponding Author \\ Ekram Abdelsalam Elfaramawy Mohamed
}

\begin{abstract}
Introduction: Cancer is the second leading cause of death in children under 15 years of age. Childhood cancers (CC) include a variety of malignant tumors. Leukemia is constituted about $30-34 \%$ of all CC. Central nervous system (CNS) cancers are the second most frequent cancers, accounting for about $27 \%$ of CC. Lymphomas represent the next most common type of CC, averaging 15\%.Patients with cancer suffer from an increased risk for common and opportunistic infections that result from intrinsic disease and anticancer therapy-induced immune deficiencies. Opportunistic enteric protozoan parasites such as Cryptosporidium spp and Microsporidia may cause severe diarrhoea, morbidity and even mortality among immunocompromised patients. Cryptosporidiosis has been reported in both immunocompetent and immunocompromised patients and it has three major clinical presentations, including; asymptomatic carriage, acute diarrhea, and persistent diarrhea.

Subjects: The study will be conducted on children aged 6 months - 16 years. It will include the following: 166 subjects, 83 immunocompromised children with diarrhea presenting to Alexandria University Children's Hospital at Elshatby and 83 age matched control cases.

Methods: Collection of Stool samples, Stool examination by Modified Ziehl - Neelsen stain for the diagnosis of C. parvum and Copro-antigen detection test using Rida Screen cryptosporidium ELISA kit.

Results: Cryptosporidium infestation was significantly more frequent in studied patients suffering from malignancy as compared with control group.

Conclusion: children with cancer are more likely to suffer from severe gastrointestinal manifestations due to the disease itself or infectious and non-infectious complications of chemotherapeutic treatment. Cryptosporidium infection should be considered in cases of severe or prolonged watery diarrhea in children with cancer which necessitates appropriate management to decrease its complications.
\end{abstract}

Keywords: Cancer, opportunistic infections, Cryptosporidiosis, ELISA.

\section{Introduction}

Cancer is the second leading cause of death in children under 15 years of age. Childhood cancers (CC) include a variety of malignant tumors.
Annual incidence worldwide is approximately 160,000 cases per year, whereas mortality rates average $90,000^{(1)}$. 
Leukemia is constituted in about $30-34 \%$ of all $\mathrm{CC}^{(1)}$. Acute lymphoblastic leukemia (ALL) and acute myeloblastic leukemia (AML) are the two most frequent types of leukemia found in children (1). The former occurs approximately five times more frequently than the latter, and accounts for approximately $75 \%$ of all childhood leukemia cases ${ }^{(1)}$. Central nervous system (CNS) cancers are the second most frequentcancers, accounting for about $27 \%$ of $\mathrm{CC}^{(2)}$ Lymphomas represent the next most common type of CC, averaging $15 \%{ }^{(3)}$. Neuroblastoma is diagnosed in $7 \%$ of the CC cases, nephroblastoma (Wilms) tumors occurs in $5 \%$ and retinoblastoma in $3 \% \mathrm{CC}$ cases ${ }^{(2)}$.

Interactions between cancer and the immune system are complex. It is clear that cancer patients display varying degrees of immunosuppression at the time of presentation, prior to initiation of antineoplastic therapy. This immunosuppression is subsequently exacerbated by prolonged and intensive chemotherapy administered with or without corticosteroids. In addition, patients with previously untreated Hodgkin's disease frequently have impaired lymphocyte proliferation to a variety of antigens, and patients with Burkitt's lymphoma have been reported to show variable levels of lymphocyte depletion which relate to the stage of disease. Even patients with sarcoma, a disease not classically associated with immunesuppression, occasionally show reduced peripheral blood T-cell populations at the time of presentation.

By suppressing the body's defensive immune functions, all immunosuppressive regimens can lead to increased rates of systemic or localized infections including those of the GI tractopportunistic enteric protozoan parasites such as Cryptosporidium spp and Microsporidia that may cause severe diarrhoea, morbidity and even mortality among immunocompromised patients. Cryptosporidium spp. infection may cause severe clinical gastrointestinal disorders, especially in people with malignancies, patients with solidorgan transplants, and hemodialysis patients.
Transmission of the parasite occurs via the fecooral route, by ingestion of contaminated water or food, person-to-person and animal-to-human transmission $^{(4)}$.

In immunocompetent hosts infection is often asymptomatic or mild to moderate and selflimited ${ }^{(5)}$. However, in immunodeficient hosts such as patients with HIV/AIDS, congenital immunodeficiencies, malignancies and transplant recipients, infection can result in persistent, debilitating and possibly fatal diarrhea and wasting ${ }^{(5)}$.

Diagnosis of cryptosporidiosis can be done by:1Detection of clinical manifestations as persistent, debilitating diarrhea ,abdominal cramp ,nausea or vomiting, mild fever, anorexia, malaise and fatigue and weight loss2- Examination of stool samples. As the cysts of Cryptosporidium, are very small, a staining procedure is required to detect them. A number of different staining techniques have been developed such as the ZiehlNeelsen stain and modified Kinyoun's stain are commonly used. Enzyme-linked immunosorbent assay (ELISA) also detects Cryptosporidium oocyst antigen in human fecal specimens . ${ }^{(6)}$

General precautions against the infection include hand washing prior to eating or preparing food and after contact with animals and washing of fruits and vegetables prior to consumption. Immunocompromised patients are also advised to boil all drinking water to reduce the risk of infection ${ }^{(7)}$

Nitazoxanide and paromomycin are the only drugs which when examined in controlled clinical trials showed some efficacy in the resolution of the infection ${ }^{(8)}$.Supportive management including rehydration therapy, electrolyte replacement and antimotility agents will remain the main treatment strategies until better drugs emerge ${ }^{(8)}$.

\section{Aim of the Study}

The aim of the work was to assess the frequency of cryptosporidiosis in immunocompromised children with diarrhea in comparison to normal 
children and study the course of the disease in both groups of children.

\section{Materials and Methods \\ Study settings}

Study was conducted in Pediatric Department at Alexandria University Children's Hospital.

\section{Target Population}

The study will be conducted on children 6 months - 16 years of age. They will include the following: 166 subjects, 83 immunocompromised children with diarrhea presenting to Alexandria University Children's Hospital at Elshatby and 83 age matched control cases.

\section{Inclusion criteria}

83 children with solid tumors and hematological malignancies of different stages and risk classifications with acute or chronic diarrhea admitted to hospital and attending out patients clinics as an example of immunocompromised children

83 age matched children with no evidence of malnutrition or of any disease affecting the immune system presenting with acute or chronic diarrhea admitted to hospital and out patients clinics

\section{Exclusion Criteria}

Infant younger than 6 months and children older than 16 years., all patients receiving anti parasitic medication. In control group history of intake of steroids, presence of malnutrition or of any disease affecting the immune system.

\section{Study Design}

Case-control cross sectional study.

\section{Collected Data}

Age, sex, Residency, Animal contact, drinking water source, type of diarrhea and type of malignancy.

Methods: Collection of Stool samples, Stool examination by Modified Ziehl - Neelsen stain for the diagnosis of $\mathrm{C}$. parvum and Copro-antigen detection test using Rida Screen cryptosporidium ELISA kit.

\section{Results}

The present study included $47(56.6 \%)$ and 37 $(44.6 \%)$ males, $36(43.4 \%)$ and $46(55.4 \%)$ females for cases and control groups respectively there were no statistical significant differences between the two studied groups regarding sex and age. Cryptosporidium infestation was significantly more frequent in studied cases compared to control group. Also study shows no statistically significant correlation exists between infestation with cryptosporidium and type of malignancy in cases and there was no significant correlation exists between infestation with cryptosporidium and sex, age, drinking water source, contact with animal and birds and residency of cases and control group.

Table (1): Comparison between the group I (cases) and group II (control) according to result of ELISA test for cryptosporidium.

\begin{tabular}{|l|c|c|c|c|c|c|}
\hline \multirow{2}{*}{ Crypto } & \multicolumn{2}{|c|}{$\begin{array}{c}\text { Cases } \\
(\mathrm{n}=83)\end{array}$} & \multicolumn{2}{c|}{$\begin{array}{c}\text { Control } \\
(\mathrm{n}=83)\end{array}$} & \multirow{2}{*}{$\chi^{2}$} & $\mathrm{p}$ \\
\cline { 2 - 5 } & No. & $\%$ & No. & $\%$ & & \\
\hline & 70 & 84.3 & 78 & 94.0 & & \\
No & 13 & 15.7 & 5 & 6.0 & $3.988^{*}$ & $0.046^{*}$ \\
Yes & 13 & & \\
\hline
\end{tabular}

$\chi^{2}$ value for Chi square test for comparing between the two groups

*:Statistically significant at $\mathrm{p} \leq 0.05$

Table (1) shows the result of ELISA test for cryptosporidium in stool of group I (cases) and group II (control). Cryptosporidium infestation was significantly more frequent in studied cases as compared with control group.

Table (2) Relation between between infestation with cryptosporidium and GIT symptoms in group I (cases) $(\mathrm{n}=83)$.

\begin{tabular}{||l|c|c|c|c|c|c||}
\hline \multirow{2}{*}{ GIT Symptoms } & \multicolumn{4}{|c|}{ Crypto } & \multirow{2}{*}{} & \multirow{2}{*}{\begin{tabular}{c} 
Yes \\
No \\
\cline { 2 - 5 }
\end{tabular}} \\
\cline { 2 - 5 } & No. & $\%$ & No. & $\%$ & & ${ }^{2} \mathrm{p}$ \\
\hline Watery diarrhea & 17 & 24.3 & 12 & 92.3 & $22.317^{*}$ & $<0.001^{*}$ \\
Loose diarrhea & 53 & 75.7 & 1 & 7.7 & $22.317^{*}$ & $<0.001^{*}$ \\
Dysentry & 0 & 0.0 & 0 & 0.0 & - & - \\
Abdominal pain & 32 & 45.7 & 13 & 100.0 & $13.017^{*}$ & $<0.001^{*}$ \\
Anorexia & 11 & 15.7 & 3 & 23.1 & 0.424 & 0.686 \\
Vomiting & 17 & 24.3 & 2 & 15.4 & 0.492 & 0.723 \\
Fever & 19 & 27.1 & 5 & 38.5 & 0.683 & 0.507 \\
\hline
\end{tabular}

$\chi^{2}$ value for Chi square test for comparing between the two groups

FE: Fisher Exact for Chi square test

*: Statistically significant at $\mathrm{p} \leq 0.05$ 
Table (2) shows that significant correlation exists between infestation with cryptosporidium and watery diarrhea and abdominal pain in studied cases $(\mathrm{p}<0.001)$.

Table (3): Relation between infestation with cryptosporidium and the type of malignancy in cases group $(\mathrm{n}=83)$

\begin{tabular}{|c|c|c|c|c|c|c|}
\hline \multirow{3}{*}{$\begin{array}{ll}\text { Type } & \text { of } \\
\text { malignancy }\end{array}$} & \multicolumn{4}{|c|}{ Crypto } & \multirow{3}{*}{$\chi^{2}$} & \multirow{3}{*}{${ }^{\mathrm{MC}} \mathrm{p}$} \\
\hline & \multicolumn{2}{|c|}{$\begin{array}{c}\text { No } \\
(\mathrm{n}=70)\end{array}$} & \multicolumn{2}{|c|}{$\begin{array}{c}\text { Yes } \\
(\mathrm{n}=13)\end{array}$} & & \\
\hline & No. & $\%$ & No. & $\%$ & & \\
\hline Leukemia & 51 & 72.9 & 13 & 100.0 & & \\
\hline Lymphoma & 10 & 14.3 & 0 & 0.0 & & \\
\hline Bone tumor & 6 & 8.6 & 0 & 0.0 & 3.017 & 0.328 \\
\hline $\begin{array}{l}\text { Wilms tumor / } \\
\text { Neuroblastoma }\end{array}$ & 3 & 4.3 & 0 & 0.0 & & \\
\hline
\end{tabular}

$\chi^{2}$ value for Chi square test for comparing between the two groups

MC: Monte Carlo for Chi square test

Table (3) shows that cryptosporidium infection is most prevalent in leukemia but there isno statistically significant correlation exists between infestation with cryptosporidium and type of malignancy in cases $(\mathrm{P}=0.328)$.

\section{Discussion}

The objective of the current study was to assess the frequency of cryptosporidiosis in immunocompromised children with diarrhea in comparison to healthy children.

In the present study, no significant correlation was found between age and infection with cryptosporidium. This finding is in agreement with what was reported by Ayalew ${ }^{(9)}$. The nonsignificant variation may be attributed to the poor personal and environmental hygiene, poor water quality and the close contact of all age groups to domestic animals. On the contrary the prevalence of cryptosporidiosis is higher in younger children mostly in children aged below 5 years was reported by Mohammadi Ghaleh Bin B, Falah S, Asgharzadeh M, et al. ${ }^{(10)}$

Current study showed no statistical significant correlation between sex and infection with cryptosporidium which may indicate that both sexes have equal chance of being infected.This finding is in agreement with a study conducted by Gatei et al., ${ }^{(12)}$ found that infection rates did not vary with gender distribution. However, an opposite observation was reported by Adamu et $\mathrm{al}^{(12)}$ and Mumtaz et al., ${ }^{(13)}$ where the prevalence of C. parvum infection in males was higher than in females. In their study, they suggested that there might have been an unmeasured intrafamilial factor functioning to expose infant boys or to protect infant girls in their study population. In the present study, no significant correlation was found between animal contact and infection with cryptosporidium. In agreement with the results of the current study, a study done in North of Baghdad by Harith S. Al-Warid ${ }^{(14)}$ demonstrated that there were no significant relations between prevalence of Cryptosporidium spp and presence of domestic animals in household. However, an opposite observation was reported from Siwila et al, Phiri et al and Yu et al where the contact with domestic animals acts as a risk factor in zoonotic infection $^{(15)}$ this may be attributed to the socioeconomic correlation of the studied group.

In the present study, Cryptosporidium infestation was significantly more frequent in children with malignancies compared to healthy children. In agreement with the results of the current study, . Ferreira et $\mathrm{al}^{(16)}$ reported that among the most important protozoa that were incriminated for causing severe diarrhea in the immunosuppressed patients was Cryptosporidium parvum.

Evering and Weiss ${ }^{(18)}$ stated that children with acute leukemia seem to be most at risk from cryptosporidiosis Berenji et $\mathrm{al}^{\left({ }^{(19)}\right.}$ reported $22 \%$ incidence of cryptosporidium infection among pediatric patients with lymphohematopoietic malignancies. Domenech et al. ${ }^{(17)}$ described two cases of acute lymphoblastic leukemia who developed cryptosporidiosis with severe diarrhea during maintenance of chemotherapy. On the other hand, Al-Megrin ${ }^{(20)}$ reported much lower incidence $(17.5 \%)$ for Cryptosporidium and among immunocompromised diarrheic patients in Riyadh, Saudi Arabia. This discrepancy with their results may be due to variations in specific 
treatment, immunological state, type of diarrhea, date, residence, occupation and age of the patients.

Also present study demonstrated that the most common presentations ofcryptosporidiosis were watery diarrhea and abdominal pain and less common were dysentry, anorexia, vomiting and fever. In agreement with the results of the current study, Smith and Corcoran ${ }^{(21)}$ reported thatthe most common clinical signs of Cryptosporidium infection are profuse and watery diarrhoea, crampy abdominal pain, fever, nausea, vomiting and low grade fever. Similarly, Mbanugo and $\mathrm{Agu}^{(22)}$ in their study of cryptosporidiosis in Anambra State, Nigeria, also obtained prevalence rates of $40.0 \%$ for watery stool samples. Also Arora $^{(23)}$ reported that Cryptosporidium cause the same type of diseases in humans and animals with symptoms including non-bloody, watery diarrhea.

\section{Conclusion}

1. Children with cancer are more likely to suffer from severe gastrointestinal manifesttations due to the disease itself or infectious and non-infectious complications of chemotherapeutic treatment.

2. Cryptosporidium infection should be considered in cases of severe or prolonged watery diarrhea in children with cancer.

\section{Recommendation}

1-Cryptosporidium infection must be considered in the differential diagnosis in patients with malignancy with diarrhea in order to reduce the suffering often faced by those patients.

2-Proper public health educations on the potential sources and transmission ways of cryptosporidium infection and how to avoid it should be given. This must include how to improve some hygienic practices too; in order to reduce fecal-water contamination in the environment.

3-Local considerations play a major role and public health authorities are encouraged to provide guidance on the safety of drinking-water for the immunocompromised and on appropriate means of reducing exposure.

4- Since cryptosporidium is not tested in routine stool examination, it should be included for confirmation of diagnosis by a MZN stain and a more specific diagnostic tool, such as ELISA.

\section{References}

1. Ferlay J, Shin H-R, Bray F, Forman D, Mathers C, Parkin DM. Estimates of worldwide burden of cancer in 2008. Int $\mathbf{J}$ Cancer 2010;8(12):2893-2917.

2. Siegel R, Naishadham D, Jemal A. Cancer statistics. CA Cancer J Clin 2012;8(1):10 29.

3. Stefan DC, Jacobs P. Lymphoma: a pediatrician's perspective. Transfus Apher Sci 2010;8(2):185-87.

4. Fayer R, Morgan U, Upton SJ. Epidemiology of Cryptosporidium: transmission, detection and identification. Int J Parasitol 2000;30(12-13):1305-22.

5. Chen XM, Keithly JS, Paya CV, LaRusso NF. Current concepts cryptosporidiosis. New Engl J Med 2002;346: 1723-31.

6. Brooks, Geo. F, Butel, Janet S, Morse, Stephen A. Jawetz, Melnick, \&Adelberg's Medical Microbiology. New York Lange Medical Books McGraw Hill 2004;23: 684-5

7. Ramirez NE, Ward LA, Sreevatsan S. A review of the biology and epidemiology of cryptosporidiosis in humans and animals. Microbes and Infection 2004; 6(8):773-85.

8. Rossignol JF, M. G, Ortega-Pierres, Ronald Faye. Drug treatment and novel drug targets against giardia and cryptosporidium. Giardia and Cryptosporidium From Molecules to Disease 2009;38: 46382.

9. Ayalew D. Assessment of the association of Cryptosporidium parvum, Giardia lamblia and Entamoebahistolytica/dispar infection prevalence with drinking water source among children in Legedini, Adada 
and Legfbira, Dire-Dawa, Eastern Ethiopia. [Unpublished M.Sc. Thesis]: Department of Biology, Addis Ababa University 2006

10. MohammadiGhaleh Bin B, Falah S, Asgharzadeh M, et al. Prevalence of Cryptosporidium in children suffering from gastroenteritis in Ardabil hospitals. J Ardabil Univ Med Sci 2006;6(2):176-82.

11. Gatei W, Wamae CN, Mbae C, Waruru A, et al., Cryptosporidiosis prevalence, genotype analysis and symptoms associated with infections in children in Kenya. Am J Trop Med Hyg 2006; 75 (1): 78-82.

12. Adamu, H. Opportunistic intestinal parasitic infections in pediatric patients with diarrhea in relation to some demographic features in Addis Ababa hospitals. Master thesis, Dept. Biology, Addis Ababa University2004; 68.

13. Mumtaz S, Ahmed J, Ali L. Frequency of cryptosporidium infection in children under five years of age having diarrhea in north west of Pakistan. Afr J Biotech 2010; 9 (8): 1230-35.

14. Al-Warid, Harith S., Ihsan M. Al-Saqur, and Souhaila H. Mahmood. "Occurrence of Cryptosporidium spp among People Live in North of Baghdad." Eur J Sci Res 2012; 78.4: 539-45.

15. Siwila J, Phiri IG, Vercruysse J, Goma F, Gabriel S, Claerebout E, Geurden T. Asymptomatic cryptosporidiosis in Zambian dairy farm workers and their household members. Trans R Soc Trop Med Hyg 2007;101(7):733-4.

16. Ferreira, M.S. Infections by protozoa in immunocompromised hosts. Memorias Instituto Oswaldo Cruz 2000; 95: 159-62.

17. Domenech, C, M. Rabodonirina, N. Bleyzac, M.P. Pages and Y. Bertrand. Cryptosporidiosis in children with acute lymphoblastic leukemia on maintenance chemotherapy. J Pediatr Hematol Oncol 2011; 33: 570-72.
18. Evering, T. and L.M. Weiss. The immunology of parasite infections in immunecompromised hosts. Parasite Immunol 2006; 28: 549-65.

19. Berenji, F., N. Zabolinejad, H. Kianifar, Z. Badeii, A. Banihashem and $S$. Hiradfar. Cryptosporidium infection in pediatric patients with lymphohematopoietic malignancies. Iran $\mathrm{J}$ Pediatr 2007;3: 247-51.

20. Al-Megrin, W.A.I. Intestinal parasites infection among immunocompromised patients in Riyadh, Saudi Arabia. Pak J BiolSci 2010; 13: 390-94.

21. Smith, H.V and Corcoran, G.D . New drugs and treatment for cryptosporidiosis. Current Opinion in Infectious Disease 2004;17:557-64.

22. Mbanugo, J.I. and Agu, V.C. Prevalence of Crptosporidiumparvum infections in children, aged 0-15years, in Anambra State, Nigeria. Nig. J. Parasitol 2006; 26:15.

23. Arora DR, Arora B. Medical Parasitology. CBS Publishers India 2009;2:90-95. 